\title{
A preliminary systematic search for red-clump stars in Galactic open clusters based on 2MASS data
}

\author{
Xi Zhang, ${ }^{1}$ Li Chen, ${ }^{2}$ and Zhongmu $\mathrm{Li}^{1}$ \\ ${ }^{1}$ Institute of Astronomy and the History of Science and Technology, Dali University, \\ Dali 671003, China \\ email: xizhang1266@gmail.com, zhongmu.li@gmail.com \\ ${ }^{2}$ Shanghai Astronomical Observatory, Chinese Academy of Sciences, 80 Nandan Road, \\ Shanghai 200030, China \\ email: chenli@shao.ac.cn
}

\begin{abstract}
Red-clump (RC) giants are intermediate-age, core-helium-burning stars. The RC can be used as a standard candle. In particular, the small variance of the RC's $K$-band intrinsic luminosity and its weak dependence on chemical composition and age make it an extremely useful distance indicator. In this paper, we use 2MAss data to search for RC stars in a sample of 60 Galactic open clusters with known reddening, ages, and distances, and obtain an average value for the RC's absolute $K_{\mathrm{s}}$-band magnitude, $M_{K_{\mathrm{s}}}=-1.72 \pm 0.17 \mathrm{mag}$.
\end{abstract}

Keywords. open clusters and associations: general, distance scale

\section{Selection of red-clump stars}

Red-clump (RC) giants are intermediate-age, core-helium-burning stars. The RC can be used as a standard candle. In particular, the RC's intrinsic $K$-band luminosity exhibits a small variance and only a weak dependence on chemical composition and age, which makes it an extremely useful distance indicator.

Van Helshoecht \& Groenewegen (2007) investigated how the RC's $K$-band magnitude, $M_{K}(\mathrm{RC})$, depends on age and metallicity, using 2MAss infrared data for a sample of 24 open clusters with known distances. Here, also based on 2MASs data, we searched for RC stars in a much larger sample of 60 Galactic open clusters with known reddening, ages, and distances, and obtained an average value for the RC's absolute $K_{\mathrm{s}}$-band magnitude.

We took a search radius of 60 arcmin around the center of our sample clusters, and adopted as the cluster radius the distance to the cluster center where the stellar density is $120 \%$ of the field-star density: see Fig. 1.

The RC has the advantage of being easily recognizable in the color-magnitude diagrams (CMDs) of most open clusters. Based on the CMDs of 60 sample clusters using 2MAss $J H K_{\mathrm{s}}$ data, we searched for possible RC members in each of our sample clusters by visual inspection. We focussed on their compact locus in the CMDs, in the knowledge that it is a highly populated region in CMD space that is brighter than the main sequence. To select RC stars in the CMD of a given cluster, a fixed-size box of 0.2 mag in color and 0.8 mag in magnitude was used (Van Helshoecht \& Groenewegen 2007): see, for an example, Fig. 2. 


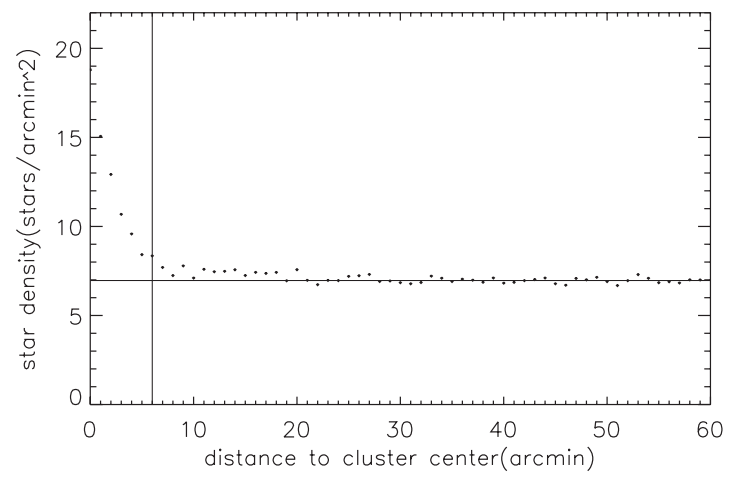

Figure 1. Example of cluster-radius estimation for the open cluster Haffner 10. The stellar density is plotted as a function of distance to the cluster center. We define the cluster radius as the distance to the cluster center where the stellar density is $120 \%$ of the field-star density. Here, a radius of $6^{\prime}$ is adopted.
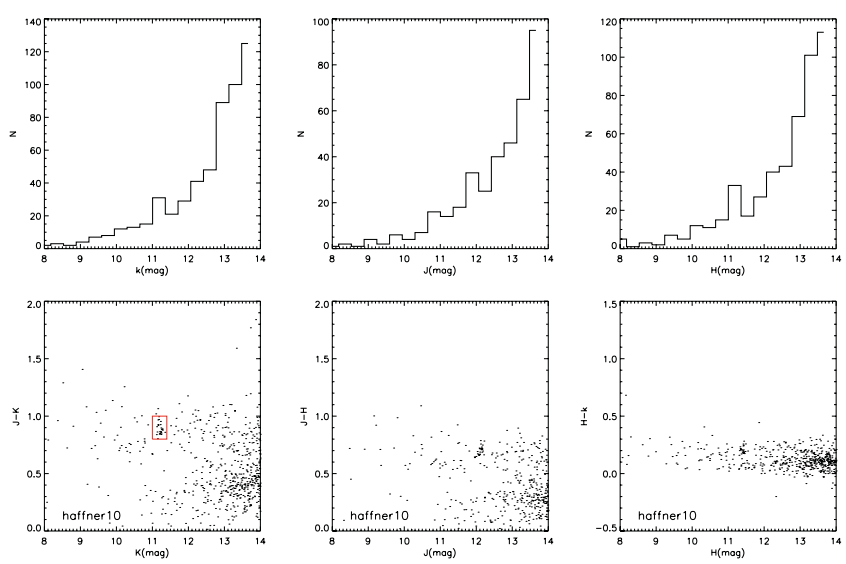

Figure 2. (top) $J_{-}, H_{-}$, and $K_{\mathrm{s}}$-band luminosity functions of Haffner 10 within a cluster radius of $6^{\prime}$. (bottom) $\left(J-K_{\mathrm{s}}\right)$ versus $K_{\mathrm{s}},(J-H)$ versus $J$, and $\left(H-K_{\mathrm{s}}\right)$ versus $H$ color-magnitude diagrams for the same area.

\section{Results and Discussion}

After selecting possible RC members from the cluster regions, the median apparent $K_{\mathrm{s}}$ magnitudes of the stars within the fixed box were calculated. Using the relations provided by Cardelli et al. (1989), in particular $R_{V}=3.1, A_{K}=0.11 A_{V}$, and $M_{K_{\mathrm{s}}}=$ $m_{K_{\mathrm{s}}}-(m-M)_{V}-A_{K}$, we calculated the median $K_{\mathrm{s}}$-band absolute magnitude, $M_{K_{\mathrm{s}}}$, of the RCs for all clusters (see Table 1) and obtained an average value for our 60 sample clusters of $M_{K_{\mathrm{s}}}=-1.72 \pm 0.17 \mathrm{mag}$. In Table 1, the first 24 sample open clusters are the same as those in Van Helshoecht \& Groenewegen (2007); the reddening and $(m-M)_{V}$ data are from Twarog et al. (1997) and Chen et al. (2003).

\section{References}

Chen, L., Hou, J. L., \& Wang, J. J. 2003, AJ, 125, 1397

Twarog, B. A., Ashman, K. M., \& Anthony-Twarog, B. J. 1997, AJ, 114, 2556

Van Helshoecht, V. \& Groenewegen, M. A. T.. 2007, A\&SA, 463, 559 
Table 1. $K_{\mathrm{s}}$-band absolute magnitude of 60 open clusters.

\begin{tabular}{|c|c|c|c|c|}
\hline Cluster & $\begin{array}{c}E(B-V) \\
\quad(\mathrm{mag})\end{array}$ & $\begin{array}{c}(m-M)_{V} \\
(\mathrm{mag})\end{array}$ & $\begin{array}{c}M_{K_{\mathrm{s}}}(\mathrm{RC}) \\
(\mathrm{mag})\end{array}$ & $\begin{array}{c}\sigma\left[M_{K_{\mathrm{s}}}(\mathrm{RC})\right. \\
(\mathrm{mag})\end{array}$ \\
\hline BE 39 & 0.11 & 13.50 & -1.98 & 0.23 \\
\hline IC 4651 & 0.11 & 10.25 & -2.20 & 0.14 \\
\hline MEL 66 & 0.14 & 13.95 & -2.21 & 0.15 \\
\hline NGC 188 & 0.09 & 11.35 & -1.95 & 0.16 \\
\hline NGC 752 & 0.04 & 8.35 & -1.32 & 0.18 \\
\hline NGC 1817 & 0.26 & 12.15 & -2.68 & 0.17 \\
\hline NGC 2099 & 0.27 & 11.55 & -2.96 & 0.22 \\
\hline NGC 2204 & 0.08 & 13.30 & -1.90 & 0.21 \\
\hline NGC 2243 & 0.06 & 13.45 & -2.09 & 0.18 \\
\hline NGC 2360 & 0.09 & 10.35 & -1.49 & 0.20 \\
\hline NGC 2420 & 0.05 & 12.10 & -1.88 & 0.09 \\
\hline NGC 2477 & 0.23 & 11.55 & -2.10 & 0.35 \\
\hline NGC 2506 & 0.05 & 12.60 & -1.77 & 0.37 \\
\hline NGC 2527 & 0.09 & 9.30 & -2.03 & 0.07 \\
\hline NGC 2682 & 0.04 & 9.80 & -1.85 & 0.02 \\
\hline NGC 3680 & 0.05 & 10.25 & -2.07 & 0.05 \\
\hline NGC 3960 & 0.29 & 12.15 & -2.06 & 0.08 \\
\hline NGC 5822 & 0.14 & 10.00 & -2.12 & 0.30 \\
\hline NGC 6134 & 0.35 & 11.10 & -2.18 & 0.20 \\
\hline NGC 6633 & 0.16 & 8.35 & -2.16 & 0.18 \\
\hline NGC 6791 & 0.15 & 13.40 & -1.92 & 0.22 \\
\hline NGC 6819 & 0.16 & 12.44 & -2.15 & 0.26 \\
\hline NGC 7789 & 0.27 & 12.45 & -2.51 & 0.27 \\
\hline TOM 2 & 0.30 & 15.60 & -2.60 & 0.23 \\
\hline Berk 21 & 0.76 & 13.49 & -1.16 & 0.11 \\
\hline Berk 24 & 0.40 & 13.36 & -1.34 & 0.37 \\
\hline Berk 30 & 0.50 & 13.40 & -2.75 & 0.12 \\
\hline Berk 44 & 1.40 & 11.28 & -0.55 & 0.13 \\
\hline Berk 66 & 1.25 & 13.58 & -1.54 & 0.09 \\
\hline Berk 78 & 0.01 & 13.41 & -3.20 & 0.11 \\
\hline Collinder 74 & 0.38 & 12.00 & -1.63 & 0.11 \\
\hline Collinder 110 & 0.50 & 11.45 & -1.35 & 0.15 \\
\hline Dolidze 38 & 0.15 & 10.40 & -1.09 & 0.07 \\
\hline Haffner 10 & 0.52 & 13.60 & -2.58 & 0.08 \\
\hline IC 166 & 0.18 & 12.99 & -0.92 & 0.66 \\
\hline King 5 & 0.00 & 11.39 & -0.79 & 0.21 \\
\hline NGC 1241 & 0.25 & 13.03 & -1.52 & 0.16 \\
\hline NGC 1245 & 0.30 & 12.29 & -1.13 & 0.32 \\
\hline NGC 2158 & 0.36 & 13.35 & -2.16 & 0.22 \\
\hline NGC 2180 & 0.00 & 9.80 & -2.33 & 0.16 \\
\hline NGC 2192 & 0.00 & 12.68 & -0.92 & 0.16 \\
\hline NGC 2194 & 0.38 & 12.89 & -2.42 & 0.31 \\
\hline NGC 2236 & 0.48 & 12.33 & -1.91 & 0.27 \\
\hline NGC 2266 & 0.10 & 11.90 & -1.24 & 0.20 \\
\hline NGC 2304 & 0.10 & 13.01 & -2.19 & 0.19 \\
\hline NGC 2355 & 0.12 & 11.71 & -1.64 & 0.13 \\
\hline NGC 2423 & 0.10 & 9.42 & -0.75 & 0.14 \\
\hline NGC 2425 & 0.21 & 12.75 & -1.87 & 0.15 \\
\hline NGC 2479 & 0.10 & 10.40 & -0.76 & 0.11 \\
\hline NGC 2533 & 0.05 & 12.64 & -1.89 & 0.10 \\
\hline NGC 2627 & 0.09 & 11.54 & -2.06 & 0.12 \\
\hline NGC 2660 & 0.31 & 12.26 & -1.32 & 0.24 \\
\hline NGC 6728 & 0.15 & 10.00 & -0.94 & 0.13 \\
\hline NGC 6802 & 0.35 & 10.25 & -0.62 & 0.20 \\
\hline NGC 6939 & 0.33 & 11.28 & -1.53 & 0.18 \\
\hline NGC 6940 & 0.21 & 9.43 & -0.65 & 0.20 \\
\hline NGC 7044 & 0.59 & 12.50 & -1.26 & 0.41 \\
\hline Pismis 2 & 0.05 & 12.60 & -1.21 & 0.05 \\
\hline Pismis 15 & $0053{ }_{b y}$ & versity Press 12. & -1.66 & 0.11 \\
\hline TOM 1 & $0.30^{\circ}$ & 11.71 & -1.08 & 0.11 \\
\hline
\end{tabular}

